\title{
Nuclear Electric Quadrapole Moments (Q) in ${ }^{58} \mathrm{Ni}$
}

\author{
Huda A. Ruzuqy, Firas Z. Majeed \\ Department of Physics, College of Science, University of Baghdad, Baghdad, Iraq
}

Email address:

hudaphy.2018@gmail.com (H. A. Ruzuqy)

\section{To cite this article:}

Huda A. Ruzuqy, Firas Z. Majeed. Nuclear Electric Quadrapole Moments (Q) in ${ }^{58}$ Ni. American Journal of Physics and Applications. Vol. 6, No. 4, 2018, pp. 80-84. doi: 10.11648/j.ajpa.20180604.11

Received: March 12, 2018; Accepted: April 10, 2018; Published: July 26, 2018

\begin{abstract}
Nuclear Electric quadrapole moments $Q$ in ${ }^{58} \mathrm{Ni}$ for some selected levels have been investigated and calculated through Nuclear shell model and considering of ${ }^{56} \mathrm{Ni}$ as an inert core with two active neutrons in a model space $\left(2 \mathrm{p}_{3 / 2}, 1 \mathrm{f}_{5 / 2}\right.$ and $2 \mathrm{p}_{1 / 2}$ ) and the configuration mixing of the original states is also done. F5Pvh interaction has been utilized as a two body interaction to generate model space vectors with harmonic oscillator potential as a single particle wave function. OXBASH code is used to carry this calculations and the program of Core, Valence, Tassie (CVT) written in FORTRAN go language to calculate the Electric quadrapole moments between excited states themselves. All of these calculations have been carried through model space vectors only. One body density matrix elements (OBDM) for ground and Excited states is calculated in order to carry the calculations using single particle Transition matrix elements between excited states theme selves.
\end{abstract}

Keywords: Shell Model, E2, Q, ${ }^{58} \mathrm{Ni}$

\section{Introduction}

A large part of the knowledge of nuclei is obtained from the study of electromagnetic transitions, since the electromagnetic interaction is well understood, in contrast with the nuclear forces. It is, for example, the main source of information about the spin assignments of nuclear states. The nuclear multipole moments and the transition rates for the various multipole radiations can be calculated theoretically, once the nuclear wave functions are known.

We shall not give a complete derivation of the required electromagnetic transition operators. Instead only some of the most important steps that lead to explicit expressions for these operators will be summarized. For example, [Blatt and Weisskopf (1952), Jackson (1962), Morse and Feshbach (1953) and Roy and Nigam (1967).

Some of the basic equations related to the electromagnetic interaction are summarized. A derivation of the operators that will be used later to calculate electromagnetic transition and moments, Centre of mass corrections are treated. Measurable quantities such as reduced transition rates, lifetimes, branching and mixing ratios are defined. The much used Weisskopf single particle estimates of transition strengths are derived. It is shown that the isospin formalism allows us to express transition strengths in terms of isoscalar and isovector contributions. This separation can be used for example, to correlate transition rates in isobaric mass multiplets [1].

The nuclear quadrupole moments vary widely in magnitude. In particular the nuclei in the mass regions $150<$ $\mathrm{A}<190$ and $\mathrm{A}>225$ possess large permanent deformations. Light nuclei usually can be considered as to consist of a spherical core with a small number of extra nucleons. In such a picture the nuclear quadrupole moment derives completely from the extra nucleons. The extra nucleons may be coupled to pairs $\mathrm{j}^{2}$ with $\mathrm{J}=0$ and then, in this extreme single-particle model, the quadrupole moment is due to the last odd proton [1].

The Nucleus ${ }^{58} \mathrm{Ni}$ Nickel $(\mathrm{Ni})$ possesses five stable isotopes including ${ }^{58} \mathrm{Ni},{ }^{60} \mathrm{Ni},{ }^{61} \mathrm{Ni},{ }^{62} \mathrm{Ni}$ and ${ }^{64} \mathrm{Ni}$. In addition, 27 radioactive isotopes have been discovered ranging from ${ }^{48} \mathrm{Ni}$ to ${ }^{79} \mathrm{Ni}$, some of them have short-half lives; others have long-half lives. The longest- half lives is ${ }^{59} \mathrm{Ni}$ with a half-life of $7.6 \times 10+4$ years. Most of them are under a minute or a second. The least unstable is ${ }^{79} \mathrm{Ni}$ with a half-life of $635 \times 10-9$ s. $[2,3]$. The nucleus Ni 58 has 28 protons and 30 neutrons, two neutrons play essential role in the model space shell, outer the closed shell when the inert core ${ }^{56} \mathrm{Ni}$ is under consideration.

Wang and Ren (2005)[4] systematically investigated the elastic electron scattering on both stable and unstable nuclei 
with the relativistic eikonal approximation, where the charge density distributions of nuclei were from the self-consistent relativistic mean field model. Calculations had shown that the relativistic eikonal approximation can reproduce the experimental data of electron scattering on nuclei ranging from the light region, such as ${ }^{12} \mathrm{C}$, to the heavy region, such as ${ }^{208} \mathrm{~Pb}$. This was the systematic test of the relativistic eikonal approximation for elastic electron scattering for both light and heavy nuclei, including the calculated charge form factors for ${ }^{48} \mathrm{Ni},{ }^{56} \mathrm{Ni},{ }^{58} \mathrm{Ni},{ }^{64} \mathrm{Ni},{ }^{68} \mathrm{Ni},{ }^{74} \mathrm{Ni}$ and ${ }^{78} \mathrm{Ni}$ isotopes.

Bespalova et al. (2010) [5] studied the experimental single-particle energies and occupation probabilities for neutron states near the Fermi energy in ${ }^{58,60,62,64} \mathrm{Ni}$ nuclei which had been obtained from joint evaluation of the data on nucleon stripping and pickup reactions on the same nucleus. The resulting data were analyzed within a mean-field model with dispersive optical-model potential. Good agreement was obtained between the calculated and experimental singleparticle energies of the subshells.

Brown et al. (2014) [6] measured high-precision reduced electric-quadrupole transition probabilities B $\left(\mathrm{E} 2 ; 0^{+}{ }_{1} \rightarrow 2^{+}{ }_{1}\right)$ from single-step coulomb excitation of semi-magnetic ${ }^{58,60,62,64} \mathrm{Ni}(\mathrm{Z}=28)$ beams at $1.8 \mathrm{Mev}$ per nucleon on a natural carbon target. The energy loss of the nickel beams through the carbon target were directly measured with a zero-degree Bragg detector and the absolute $\mathrm{B}(\mathrm{E} 2)$ values were normalized by Rutherford scattering. The B (E2) values disagree with recent lifetime studies that employed the Doppler-shift attenuation method. The high-precision B(E2) values reveal an asymmetry about ${ }^{62} \mathrm{Ni}$, midshell between $\mathrm{N}=28$ and 40 , with larger values towards ${ }^{56} \mathrm{Ni}(\mathrm{Z}=\mathrm{N}=28)$. The experimental B (E2) values were compared with shellmodel calculations in the full pf model space and the results indicated a soft ${ }^{56} \mathrm{Ni}$ core.

Yao et al. (2014)[7] calculated elastic and in elastic form factor and for the transition from the ground state to $\mathrm{J}+1$ ( $\mathrm{L}$ $=\mathrm{J}=2,4)$ state in $58-68 \mathrm{Ni}$ and $24 \mathrm{Mg}$, the starting point of method was a set of Hartree-Fock-Bogoliubov wave functions generated with a constraint on the axial quadrupole moment and using a Skyrme energy density functional. Correlations beyond the mean field were introduced by projecting mean-field wave functions on angular-momentum and particle number by mixing the symmetry restored wave functions.

\section{Theory}

The nuclei are assumed to have a spherical shape. This is a good approximation for nuclei that have magic numbers of neutrons or protons: $2,8,20,28,50,82$ and 126 . These numbers come from the shell structure of the nucleus. Nuclei with magic numbers of neutrons or protons have a "closed shell" that encourages a spherical shape. Nuclei with $Z$ or $N$ far from a magic number are generally deformed. The simplest deformations are so-called quadrupole deformations where the nucleus can take either a prolate shape (rugby ball) or an oblate shape (cushion). The electric quadrupole moment $Q$ is considered as a criterion of the deviation of the electric charges of the nucleus from the spherical shape or spherical distribution.

The electric quadrupole moment operator is given by [8]:

$$
\hat{Q}=\int d r \hat{\rho}\left(\vec{r}, t_{Z}\right)\left(3 z^{2}-r^{2}\right)
$$

Where $z$ and $r$ are the position coordinates of $k$ th nucleon and $r$ is given in Cartesian coordinates as $r=x^{2}+y^{2}+z^{2}$.

$$
\begin{gathered}
\hat{O}(E J M)=\sum_{k=1}^{A} e_{t_{Z}}(k) r^{J}(k) Y_{J M}\left(\Omega_{k}\right) \\
\hat{Q}=\sqrt{\frac{16 \pi}{5}} \sum_{k=1}^{A} e_{t_{Z}}(k) r^{2}(k) Y_{20}\left(\Omega_{k}\right)
\end{gathered}
$$

After substituting equation (2) with $J=2$ into equation(3), one can obtain:

$$
\widehat{\mathrm{Q}}=\sqrt{\frac{16 \pi}{5}} \widehat{\mathrm{O}}(\mathrm{E} 20)
$$

The initial and final states of the nucleus can be written as:

$$
\begin{aligned}
& \left|J_{i} M_{i} T_{i} T_{z i}\right\rangle \equiv \text { initial state } \\
& \left|J_{f} M_{f} T_{f} T_{z f}\right\rangle \equiv \text { final state }
\end{aligned}
$$

The matrix element of the electric quadrupole operator of equation (4), between the initial and final states of the nucleus, is given by:

$$
\begin{array}{r}
\left\langle J_{\mathrm{f}} \mathrm{M}_{\mathrm{f}} \mathrm{T}_{\mathrm{f}} \mathrm{T}_{\mathrm{Z}_{\mathrm{f}}}|\widehat{\mathrm{Q}}| \mathrm{J}_{\mathrm{i}} \mathrm{M}_{\mathrm{i}} \mathrm{T}_{\mathrm{i}} \mathrm{T}_{\mathrm{Z}_{\mathrm{i}}}\right\rangle= \\
\sqrt{\frac{16 \pi}{5}}\left\langle\mathrm{~J}_{\mathrm{f}} \mathrm{M}_{\mathrm{f}} \mathrm{T}_{\mathrm{f}} \mathrm{T}_{\mathrm{Z}_{\mathrm{f}}}|\widehat{\mathrm{O}}(\mathrm{E} 20)| \mathrm{J}_{\mathrm{i}} \mathrm{M}_{\mathrm{i}} \mathrm{T}_{\mathrm{i}} \mathrm{T}_{\mathrm{Z}_{\mathrm{i}}}\right\rangle \quad \text { (5) }
\end{array}
$$

The matrix element of the electric transition operator in the above equation can be reduced by using Wigner-Eckart theorem as:

$$
\begin{gathered}
\left\langle J_{f} M_{f} T_{f} T_{Z_{f}}|\hat{O}(E 20)| J_{i} M_{i} T_{i} T_{Z_{i}}\right\rangle=(-1)^{J_{f}-M_{f}}\left(\begin{array}{ccc}
J_{f} & 2 & J_{i} \\
-M_{f} & 0 & M_{i}
\end{array}\right) \\
\times\left\langle J_{f} T_{f} T_{Z_{f}}|| \hat{O}(E 2)|| J_{i} T_{i} T_{Z_{i}}\right\rangle
\end{gathered}
$$

In nuclear physics the quadrupole moment of a state of angular momentum $J$ is defined as the expectation value of the electric quadrupole moment operator in the state $M=J$ [1]. So, equation (5) can be simplified with the aid of equation (6)as:

$$
Q=\sqrt{\frac{16 \pi}{5}}\left(\begin{array}{ccc}
J_{f} & 2 & J_{i} \\
-J_{f} & 0 & J_{i}
\end{array}\right)\left\langle J_{f} T_{f} T_{Z_{f}}|| \hat{O}(E 2)|| J_{i} T_{i} T_{Z_{i}}\right\rangle
$$

With the use of Wigner-Eckart theorem, the reduced matrix element of equation (7) can be written as:

$$
\begin{aligned}
\left\langle J_{f} T_{f} T_{Z_{f}}|| \hat{O}(E 2)|| J_{i} T_{i} T_{Z_{i}}\right\rangle= & \sum_{T=0,1}(-1)^{T_{f}-T_{Z_{f}}}\left(\begin{array}{ccc}
T_{f} & T & T_{i} \\
-T_{Z_{f}} & 0 & T_{Z_{i}}
\end{array}\right) \\
& \times\left\langle J_{f} T_{f}|\|\hat{O}(E 2)\|| J_{i} T_{i}\right\rangle
\end{aligned}
$$

And the electric quadrupole moment of equation (7) 
becomes:

$$
\begin{aligned}
Q=\left(\begin{array}{ccc}
J_{f} & 2 & J_{i} \\
-J_{f} & 0 & J_{i}
\end{array}\right) \sqrt{\frac{16 \pi}{5}} & \sum_{T=0,1}(-1)^{T_{f}-T_{Z_{f}}}\left(\begin{array}{ccc}
T_{f} & T & T_{i} \\
-T_{Z_{f}} & 0 & T_{Z_{i}}
\end{array}\right) \\
& \times\left\langle J_{f} T_{f}\left|\left\|\hat{O}_{2 T}\right\|\right| J_{i} T_{i}\right\rangle
\end{aligned}
$$

With using Wigner-Eckart theorem [9], the reduced electric transition probability can be calculated in terms of the many-particle matrix element of the electric multipole transition operator reduced in spin-isospin as:

$$
B(E J)=\frac{1}{2 J_{i}+1}\left|\sum_{T=0,1}(-1)^{T_{f}-T_{Z_{f}}}\left(\begin{array}{ccc}
T_{f} & T & T_{i} \\
-T_{Z_{f}} & M_{T} & T_{Z_{i}}
\end{array}\right)\left\langle J_{f} T_{f}\left|\left\|\hat{O}_{J T}\right\|\right| J_{i} T_{i}\right\rangle\right|^{2}
$$

Where the reduced many-particle matrix element of the electric multipole transition operator is given $\widehat{T}_{J T}^{\eta}$ by $\hat{O}_{J T}$, as:

$$
\left\langle U_{f} T_{f}\left\|\left|\hat{O}_{J T}\right|\right\| J_{i} T_{i}\right\rangle=\sum_{\alpha, \beta} O B D M\left(J_{f}, J_{i}, \alpha, \beta\right)\left\langle\alpha\left\|\left|\hat{O}_{J T}\right|\right\| \beta\right\rangle
$$

Then, equation $(1-11)$ can be written, in spin-isospin formalism

$$
\left\langle\mathrm{J}_{\mathrm{f}} \mathrm{T}_{\mathrm{f}}\left|\left\|\widehat{0}_{\mathrm{JT}}\right\|\right| \mathrm{J}_{\mathrm{i}} \mathrm{T}_{\mathrm{i}}\right\rangle=\mathrm{e}_{\mathrm{T}} \sum_{\mathrm{j}_{\mathrm{f}} \mathrm{j}_{\mathrm{i}}} \mathrm{OBDM}\left(\mathrm{J}_{\mathrm{f}}, \mathrm{J}_{\mathrm{i}}, \mathrm{j}_{\mathrm{f}}, \mathrm{j}_{\mathrm{i}}, \mathrm{J}, \Delta \mathrm{T}\right) \sqrt{2(2 \mathrm{~T}+1)} \times\left\langle l_{f} \frac{1}{2} j_{f}\left\|Y_{J}\left(\Omega_{r}\right)\right\| l_{i} \frac{1}{2} j_{i}\right\rangle\left\langle n_{f} l_{f}\left|r^{J}\right| n_{i} l_{i}\right\rangle
$$

Also, equation (11) can be written in proton-neutron formalism as:

$$
\left\langle J_{f} T_{f}\left|\left\|\hat{O}_{J T}\right\|\right| J_{i} T_{i}\right\rangle=e_{t_{Z}} \sum_{j_{f}, j_{i}} O B D M\left(J_{f}, J_{i}, j_{f}, j_{i}, J, t_{Z}\right) \times\left\langle l_{f} \frac{1}{2} j_{f}\left\|Y_{J}\left(\Omega_{r}\right)\right\| l_{i} \frac{1}{2} j_{i}\right\rangle\left\langle n_{f} l_{f}\left|r^{J}\right| n_{i} l_{i}\right\rangle
$$

Where the reduced many-particle matrix element of the electric transition operator can be calculated in spin-isospin formalism and in proton-neutron formalism with using equations (12) and (13), respectively.

From equations (9)and(10), one can show that the quadrupole moment is related to the reduced transition probability as:

$$
Q=\left(\begin{array}{ccc}
J_{f} & 2 & J_{i} \\
-J_{f} & 0 & J_{i}
\end{array}\right) \sqrt{\frac{16 \pi}{5}} \sqrt{\left(2 J_{i}+1\right) B(E J)}
$$

The electric quadrupole moment is to be taken in units of e. $f \mathrm{~m}^{2}$ or $e . b$.

Where $\mathrm{b}$ is the barn, $b=100 \mathrm{fm}^{2}$ [10].

The single particle quadrupole moment $Q_{\text {s.p. }}(j)$ of a nucleon in an orbit with spin $j$ depends on the radial and angular properties of the orbit as shown in the following equation [8]:

$$
Q_{\text {s.p. }}=-e_{j} \frac{2 j-1}{2 j+2}\left\langle r_{j}^{2}\right\rangle
$$

Where $\left\langle r_{j}^{2}\right\rangle$ is the mean square radius for a particle in the $\operatorname{orbit}(n, l, j)$.

\section{Result and Discussion}

The relation between a Clebsch-Gordon coefficient and 3-j symbol as the following [1].

$$
\begin{aligned}
&\left(\begin{array}{ccc}
J i & J & J f \\
M i & M & M f
\end{array}\right)=(-1)^{J f}\left(\begin{array}{ccc}
J i & J & J f \\
M i & M & M f
\end{array}\right) \\
&\left(\begin{array}{ccc}
j & 2 & j \\
-m & 0 & m
\end{array}\right)=(-1)^{j-m} \frac{3 m^{2}-j(j+1)}{\sqrt{(2 j-1) j(2 j+1)(j+1)(2 j+3)}} \delta m m^{\prime}
\end{aligned}
$$

The values of $3-\mathrm{j}$ symbol from state to the same state are clarified below.

$$
\begin{aligned}
& 1 \rightarrow 1 \\
& \text { 1) }\left(\begin{array}{ccc}
1 & 2 & 1 \\
-1 & 0 & 1
\end{array}\right) \approx 0.18 \\
& \text { 2) }\left(\begin{array}{ccc}
2 & 2 & 2 \\
-2 & 0 & 2
\end{array}\right) \approx 0.23 \\
& 3 \rightarrow 3 \\
& \text { 3) }\left(\begin{array}{ccc}
3 & 2 & 3 \\
-3 & 0 & 3
\end{array}\right) \approx 0.21 \\
& \text { 4) }\left(\begin{array}{ccc}
4 & 2 & 4 \\
-4 & 0 & 4
\end{array}\right) \approx 0.237
\end{aligned}
$$

Substitute values of the $3-\mathrm{j}$ symbol and the probabilities in the equation of Electric quadrupole moment $\mathrm{Q}$ in unit $\left(\mathrm{e} \mathrm{fm}^{2}\right)$ as shown below.

$$
Q=\left(\begin{array}{ccc}
J_{f} & 2 & J_{i} \\
-J_{f} & 0 & J_{i}
\end{array}\right) \sqrt{\frac{16 \pi}{5}} \sqrt{\left(2 J_{i}+1\right) B(E J)}
$$

Obtained the values of electric quadrupole moment (Q) as shown in the table 1 .

Table 1. Shows the transitions, the probabilities B(E2) and Electric quadrupole moment $(Q)$

\begin{tabular}{llllll}
\hline $\mathbf{K i}$ & $\mathbf{J i}$ & $\mathbf{K f}$ & $\mathbf{J f}$ & $\mathbf{B}(\mathbf{X L}) \times \mathbf{1 0}^{-\mathbf{1 3}}$ & $\mathbf{Q} \times \mathbf{1 0}^{\mathbf{- 5}} \mathbf{e} . \mathbf{f m}^{\mathbf{2}}$ \\
\hline 1 & 4 & 1 & 4 & 0.5678 & 0.05369 \\
1 & 4 & 2 & 4 & 5.381 & 0.16532 \\
2 & 4 & 2 & 4 & 5.381 & 0.16532 \\
2 & 4 & 1 & 4 & 0.5678 & 0.05369 \\
1 & 1 & 1 & 1 & 1764 & 1.3125 \\
1 & 1 & 2 & 1 & 1605 & 1.2519 \\
2 & 1 & 1 & 1 & 1764 & 1.3125 \\
2 & 1 & 2 & 1 & 1605 & 1.2519 \\
2 & 3 & 2 & 3 & 1296 & 2.0049 \\
1 & 3 & 1 & 3 & 1591 & 2.2412 \\
1 & 3 & 2 & 3 & 1296 & 2.0049 \\
\hline
\end{tabular}




\begin{tabular}{|c|c|c|c|c|c|}
\hline$\overline{\mathbf{K i}}$ & $\mathbf{J i}$ & $\overline{K f}$ & Jf & B $(\mathrm{XL}) \times 10^{-13}$ & $Q \times 10^{-5}$ e. $\mathrm{fm}^{2}$ \\
\hline 2 & 3 & 1 & 3 & 1591 & 2.2412 \\
\hline 1 & 2 & 1 & 2 & 737.4 & 1.3998 \\
\hline 1 & 2 & 2 & 2 & 1749 & 2.1559 \\
\hline 1 & 2 & 3 & 2 & 5501 & 3.8235 \\
\hline 1 & 2 & 4 & 2 & 1959 & 2.2817 \\
\hline 1 & 2 & 5 & 2 & 1233 & 1.8101 \\
\hline 2 & 2 & 1 & 2 & 737.4 & 1.3998 \\
\hline 2 & 2 & 2 & 2 & 1749 & 2.1559 \\
\hline 2 & 2 & 3 & 2 & 5501 & 3.8235 \\
\hline 2 & 2 & 4 & 2 & 1959 & 2.2817 \\
\hline 2 & 2 & 5 & 2 & 1233 & 1.8101 \\
\hline 3 & 2 & 1 & 2 & 737.4 & 1.3998 \\
\hline 3 & 2 & 2 & 2 & 1749 & 2.1559 \\
\hline 3 & 2 & 3 & 2 & 5501 & 3.8235 \\
\hline 3 & 2 & 4 & 2 & 1959 & 2.2817 \\
\hline 3 & 2 & 5 & 2 & 1233 & 1.8101 \\
\hline 4 & 2 & 1 & 2 & 737.4 & 1.3998 \\
\hline 4 & 2 & 2 & 2 & 1749 & 2.1559 \\
\hline 4 & 2 & 3 & 2 & 5501 & 3.8235 \\
\hline 4 & 2 & 4 & 2 & 1959 & 2.2817 \\
\hline 4 & 2 & 5 & 2 & 1233 & 1.8101 \\
\hline 5 & 2 & 1 & 2 & 737.4 & 1.3998 \\
\hline 5 & 2 & 2 & 2 & 1749 & 2.1559 \\
\hline 5 & 2 & 3 & 2 & 5501 & 3.8235 \\
\hline 5 & 2 & 4 & 2 & 1959 & 2.2817 \\
\hline 5 & 2 & 5 & 2 & 1233 & 1.8101 \\
\hline
\end{tabular}

The free neutron has e zero charge but the effective neutron has an effective charge. The measurement proved that the amount (negative) and the value less than one electronic charge.

There is a research and study of the measurements for the nuclear shell model and multipolarities which proved the existence of active charge of neutrons.

The values of the OBDM elements for electric quadrupole moment(Q) for transitions from state to the same state as shown in the tables from (2) to (12).

Table 2. The values of the OBDM for transition from excited state $\left(4^{+}\right)$to excited state $\left(4^{+}{ }_{1}\right)$

\begin{tabular}{lllll}
\hline $\mathbf{J i}$ & Jf & Ki & Kf & $\mathbf{L}$ \\
\hline $4^{+}$ & $4^{+}$ & 1 & 1 & 2 \\
$4^{+}$ & $4^{+}$ & 2 & 1 & 2 \\
nlj & n'lj'O $^{\prime}$ & OBDM(DT=0) & OBDM(DT=1) & \\
1f $5 / 2$ & 1f $5 / 2$ & 1.25168 & 1.02199 & \\
1f $5 / 2$ & 2p 3/2 & -0.20387 & -0.16646 & \\
2p 3/2 & 1f $5 / 2$ & 0.20387 & 0.16646 & \\
2p 3/2 & 2p 3/2 & 1.13002 & 0.92266 & \\
\hline
\end{tabular}

Table 3. The values of the OBDM for transition from excited state $\left(4^{+}\right)$to excited state $\left(4^{+}{ }_{2}\right)$.

\begin{tabular}{lllll}
\hline $\mathbf{J i}$ & $\mathbf{J f}$ & $\mathbf{K i}$ & $\mathbf{K f}$ & $\mathbf{L}$ \\
\hline $4^{+}$ & $4^{+}$ & 1 & 2 & 2 \\
$4^{+}$ & $4^{+}$ & 2 & 2 & 2 \\
nlj & n'l $\mathrm{j}^{\prime}$ & OBDM(DT=0) & OBDM(DT=1) & \\
1f $5 / 2$ & 1f $5 / 2$ & 0.03398 & 0.02774 & \\
1f $5 / 2$ & 2p $3 / 2$ & 1.47954 & 1.20804 & \\
2p $3 / 2$ & 1f $5 / 2$ & 0.02809 & 0.02294 & \\
2p $3 / 2$ & 2p $3 / 2$ & 0.15571 & 0.12714 & \\
\hline
\end{tabular}

Table 4. The values of the OBDM for transition from excited state $\left(1^{+}\right)$to excited state $\left(1^{+}{ }_{1}\right)$

\begin{tabular}{lllll}
\hline $\mathbf{J i}$ & $\mathbf{J f}$ & $\mathbf{K i}$ & $\mathbf{K f}$ & $\mathbf{L}$ \\
\hline $1^{+}$ & $1^{+}$ & 1 & 1 & 2 \\
$1^{+}$ & $1^{+}$ & 2 & 1 & 2 \\
$\mathrm{n} 1 \mathrm{j}$ & n' $1 \mathrm{j}^{+}$ & OBDM(DT=0) & OBDM(DT=1) & \\
1f $5 / 2$ & 1f $5 / 2$ & 0.37865 & 0.30916 & \\
1f $5 / 2$ & 2p $1 / 2$ & 0.31128 & 0.25416 & \\
2p $3 / 2$ & 2p $3 / 2$ & 0.25423 & 0.20758 & \\
2p $1 / 2$ & 1f $5 / 2$ & 0.31128 & 0.25416 & \\
\hline
\end{tabular}

Table 5. The values of the OBDM for transition from excited state $\left(1^{+}\right)$to excited state $\left(1^{+}\right)$.

\begin{tabular}{lllll}
\hline $\mathbf{J i}$ & $\mathbf{J f}$ & $\mathbf{K i}$ & $\mathbf{K f}$ & $\mathbf{L}$ \\
\hline $1^{+}$ & $1^{+}$ & 1 & 2 & 2 \\
$1^{+}$ & $1^{+}$ & 2 & 2 & 2 \\
nlj & n'l $\mathrm{j}^{+}$ & OBDM(DT=0) & OBDM(DT=1) & \\
1f $5 / 2$ & 1f $5 / 2$ & 0.17362 & 0.14176 & \\
1f $5 / 2$ & 2p $1 / 2$ & 0.14273 & 0.11654 & \\
2p $3 / 2$ & 2p3/2 & -0.18561 & -0.22732 & \\
2p $1 / 2$ & 1f $5 / 2$ & -0.67886 & -0.55428 & \\
\hline
\end{tabular}

Table 6. The values of the OBDM for transition from excited state $\left(3^{+}\right)$to excited state $\left(3^{+}\right)$.

\begin{tabular}{lllll}
\hline $\mathbf{J i}$ & $\mathbf{J f}$ & $\mathbf{K i}$ & $\mathbf{K f}$ & $\mathbf{L}$ \\
\hline $3^{+}$ & $3^{+}$ & 1 & 2 & 2 \\
$3^{+}$ & $3^{+}$ & 2 & 2 & 2 \\
$\mathrm{n} 1 \mathrm{j}$ & n'l $\mathrm{j}^{+}$ & OBDM(DT=0) & OBDM(DT=1) & \\
$1 \mathrm{f} 5 / 2$ & $1 \mathrm{f} 5 / 2$ & -0.05902 & -0.04819 & \\
$2 \mathrm{p} 3 / 2$ & $2 \mathrm{p} 3 / 2$ & -0.03005 & -0.02454 & \\
$2 \mathrm{p} 3 / 2$ & $2 \mathrm{p} 1 / 2$ & 0.01456 & 0.01189 & \\
$2 \mathrm{p} 1 / 2$ & 2p $3 / 2$ & 1.24043 & 1.01281 & \\
\hline
\end{tabular}

Table 7. The values of the OBDM for transition from excited state $\left(3^{+}\right)$to excited state $\left(3^{+}{ }_{1}\right)$.

\begin{tabular}{lllll}
\hline $\mathbf{J i}$ & $\mathbf{J f}$ & $\mathbf{K i}$ & $\mathbf{K f}$ & $\mathbf{L}$ \\
\hline $3^{+}$ & $3^{+}$ & 1 & 1 & 2 \\
$3^{+}$ & $3^{+}$ & 2 & 1 & 2 \\
$\mathrm{n} 1 \mathrm{j}$ & $\mathrm{n}^{\prime} \mathrm{l}^{\mathrm{j}} \mathrm{j}$ & OBDM(DT=0) & OBDM(DT=1) & \\
$1 \mathrm{f} 5 / 2$ & $1 \mathrm{f} 5 / 2$ & 0.68 & 0.55522 & \\
$2 \mathrm{p} 3 / 2$ & $2 \mathrm{p} 3 / 2$ & -0.27737 & -0.22647 & \\
$2 \mathrm{p} 3 / 2$ & $2 \mathrm{p} 1 / 2$ & 0.13439 & 0.10973 & \\
$2 \mathrm{p} 1 / 2$ & $2 \mathrm{p} 3 / 2$ & -0.13439 & 0.10973 & \\
\hline
\end{tabular}

Table 8. The values of the OBDM for transition from excited state $\left(2^{+}\right)$to excited state $\left(2^{+}{ }_{1}\right)$.

\begin{tabular}{|c|c|c|c|c|}
\hline $\mathbf{J i}$ & $\mathbf{J f}$ & $\mathbf{K i}$ & Kf & $\mathbf{L}$ \\
\hline $2^{+}$ & $2^{+}$ & 1 & 1 & 2 \\
\hline $2^{+}$ & $2^{+}$ & 2 & 1 & 2 \\
\hline $2^{+}$ & $2^{+}$ & 3 & 1 & 2 \\
\hline $2^{+}$ & $2^{+}$ & 4 & 1 & 2 \\
\hline $2^{+}$ & $2^{+}$ & 5 & 1 & 2 \\
\hline$n \mathrm{lj}$ & $n^{`} l{ }^{\prime} j$ & $\mathrm{OBDM}(\mathrm{DT}=0)$ & $\mathrm{OBDM}(\mathrm{DT}=1)$ & \\
\hline 1f $5 / 2$ & 1f $5 / 2$ & 0.10655 & 0.087 & \\
\hline 1f $5 / 2$ & $2 \mathrm{p} 3 / 2$ & 0.01591 & 0.01299 & \\
\hline 1f $5 / 2$ & $2 \mathrm{p} 1 / 2$ & 0.19479 & 0.15905 & \\
\hline $2 \mathrm{p} 3 / 2$ & if $5 / 2$ & -0.01591 & -0.01299 & \\
\hline $2 \mathrm{p} 3 / 2$ & $2 \mathrm{p} 3 / 2$ & 0.29103 & 0.23763 & \\
\hline $2 \mathrm{p} 3 / 2$ & $2 \mathrm{p} 1 / 2$ & 0.64202 & 0.5242 & \\
\hline $2 \mathrm{p} 1 / 2$ & if $5 / 2$ & 0.19479 & 0.15905 & \\
\hline $2 \mathrm{p} 1 / 2$ & $2 \mathrm{p} 3 / 2$ & -0.64202 & -0.5242 & \\
\hline
\end{tabular}


Table 9. The values of the OBDM for transition from excited state $\left(2^{+}\right)$to excited state $\left(2^{+}{ }_{2}\right)$.

\begin{tabular}{|c|c|c|c|c|}
\hline $\mathbf{J i}$ & Jf & $\mathbf{K i}$ & Kf & $\mathbf{L}$ \\
\hline $2^{+}$ & $2^{+}$ & 1 & 2 & 2 \\
\hline $2^{+}$ & $2^{+}$ & 2 & 2 & 2 \\
\hline $2^{+}$ & $2^{+}$ & 3 & 2 & 2 \\
\hline $2^{+}$ & $2^{+}$ & 4 & 2 & 2 \\
\hline $2^{+}$ & $2^{+}$ & 5 & 2 & 2 \\
\hline $\mathrm{n} 1 \mathrm{j}$ & $n^{`} l \dddot{j}$ & $\operatorname{OBDM}(\mathrm{DT}=0)$ & $\mathrm{OBDM}(\mathrm{DT}=1)$ & \\
\hline 1f $5 / 2$ & 1f $5 / 2$ & 0.06459 & 0.05274 & \\
\hline 1f $5 / 2$ & $2 \mathrm{p} 3 / 2$ & 0.48937 & 0.39957 & \\
\hline 1f $5 / 2$ & $2 \mathrm{p} 1 / 2$ & 0.3285 & 0.26822 & \\
\hline $2 \mathrm{p} 3 / 2$ & 1f $5 / 2$ & 0.29984 & 0.24482 & \\
\hline $2 \mathrm{p} 3 / 2$ & $2 \mathrm{p} 3 / 2$ & 0.08668 & 0.07077 & \\
\hline $2 \mathrm{p} 3 / 2$ & $2 \mathrm{p} 1 / 2$ & -0.35842 & -0.29264 & \\
\hline $2 \mathrm{p} 1 / 2$ & 1f $5 / 2$ & 0.08807 & 0.07191 & \\
\hline 2 p $1 / 2$ & 2 p $3 / 2$ & -0.38569 & -0.31491 & \\
\hline
\end{tabular}

Table 10. The values of the OBDM for transition from excited state $\left(2^{+}\right)$to excited state $\left(2^{+}{ }_{3}\right)$.

\begin{tabular}{lllll}
\hline $\mathbf{J i}$ & $\mathbf{J f}$ & $\mathbf{K i}$ & $\mathbf{K f}$ & $\mathbf{L}$ \\
$2^{+}$ & $2^{+}$ & 1 & 3 & 2 \\
$2^{+}$ & $2^{+}$ & 2 & 3 & 2 \\
$2^{+}$ & $2^{+}$ & 3 & 3 & 2 \\
$2^{+}$ & $2^{+}$ & 4 & 3 & 2 \\
$2^{+}$ & $2^{+}$ & 5 & 3 & 2 \\
$\mathrm{n} 1 \mathrm{j}$ & $\mathrm{n}^{+} \mathrm{j} \mathrm{j}$ & OBDM(DT=0) & OBDM(DT $=1)$ & \\
1f $5 / 2$ & 1f $5 / 2$ & 0.01666 & 0.0136 & \\
1f $5 / 2$ & 2p $3 / 2$ & 0.6325 & 0.51643 & \\
1f $5 / 2$ & 2p $1 / 2$ & 0.12163 & 0.09931 & \\
2p $3 / 2$ & 1f $5 / 2$ & -0.12799 & -0.10451 & \\
2p $3 / 2$ & 2p $3 / 2$ & -0.44736 & -0.36527 & \\
2p $3 / 2$ & 2p $1 / 2$ & 0.49116 & 0.40103 & \\
2p $1 / 2$ & 1f $5 / 2$ & -0.13202 & -0.1078 & \\
2p $1 / 2$ & $2 \mathrm{p} 3 / 2$ & 0.63697 & 0.52008 & \\
\hline
\end{tabular}

Table 11. The values of the OBDM for transition from excited state $\left(2^{+}\right)$to excited state $\left(2^{+}{ }_{4}\right)$.

\begin{tabular}{lllll}
\hline $\mathbf{J i}$ & $\mathbf{J f}$ & $\mathbf{K i}$ & $\mathbf{K f}$ & $\mathbf{L}$ \\
\hline $2^{+}$ & $2^{+}$ & 1 & 4 & 2 \\
$2^{+}$ & $2^{+}$ & 2 & 4 & 2 \\
$2^{+}$ & $2^{+}$ & 3 & 4 & 2 \\
$2^{+}$ & $2^{+}$ & 4 & 4 & 2 \\
$2^{+}$ & $2^{+}$ & 5 & 4 & 2 \\
$\mathrm{n} 1 \mathrm{j}$ & $\mathrm{n}^{+} \mathrm{j} \mathrm{j}$ & OBDM(DT & OBDM(DT $=1)$ & \\
1f $5 / 2$ & 1f $5 / 2$ & 0.15007 & 0.12253 & \\
1f $5 / 2$ & 2p $3 / 2$ & 0.15755 & 0.12864 & \\
2p $1 / 2$ & 1f $5 / 2$ & -0.44942 & -0.36695 & \\
2p $3 / 2$ & 1f $5 / 2$ & 0.10355 & 0.08455 & \\
2p $3 / 2$ & 2p $3 / 2$ & 0.2466 & 0.20134 & \\
2p $3 / 2$ & 2p $1 / 2$ & 0.01534 & 0.01253 & \\
2p $1 / 2$ & 1f $5 / 2$ & 0.03823 & 0.03122 & \\
2p $1 / 2$ & $2 \mathrm{p} 3 / 2$ & -0.38291 & -0.46896 & \\
\hline
\end{tabular}

Table 12. The values of the OBDM for transition from excited state $\left(2^{+}\right)$to excited state $\left(2^{+}{ }_{5}\right)$.

\begin{tabular}{lllll}
\hline $\mathbf{J i}$ & $\mathbf{J f}$ & $\mathbf{K i}$ & $\mathbf{K f}$ & $\mathbf{L}$ \\
\hline $2^{+}$ & $2^{+}$ & 1 & 5 & 2 \\
$2^{+}$ & $2^{+}$ & 2 & 5 & 2 \\
$2^{+}$ & $2^{+}$ & 3 & 5 & 2 \\
$2^{+}$ & $2^{+}$ & 4 & 5 & 2 \\
$2^{+}$ & $2^{+}$ & 5 & 5 & 2 \\
$\mathrm{n} 1 \mathrm{j}$ & $\mathrm{n}$ ' $\mathrm{j}$ & OBDM(DT $=0)$ & OBDM(DT=1) & \\
1f 5/2 & $1 \mathrm{f} \mathrm{5/2}$ & 0.36626 & 0.29905 & \\
1f 5/2 & 2p 3/2 & -0.30587 & -0.24974 & \\
\hline
\end{tabular}

\begin{tabular}{lllll}
\hline $\mathbf{J i}$ & Jf & Ki & Kf & $\mathbf{L}$ \\
\hline 1f $5 / 2$ & 2p $1 / 2$ & -0.12411 & -0.10134 & \\
2p 3/2 & 1f $5 / 2$ & -0.06029 & -0.04923 & \\
2p 3/2 & 2p $3 / 2$ & -0.21296 & -0.17388 & \\
2p 3/2 & 2p $1 / 2$ & -0.10789 & -0.08809 & \\
2p $1 / 2$ & 1f $5 / 2$ & 0.27101 & 0.22128 & \\
2p $1 / 2$ & 2p 3/2 & 0.2939 & 0.23997 & \\
\hline
\end{tabular}

\section{Conclusion}

Very weak values of Electric Quadrapole moment $(\mathrm{Q})$ are generated from ${ }^{58} \mathrm{Ni}$ due to its active particles (Neutron) which are neutral particles $\left(\mathrm{e}_{\mathrm{n}}=0\right)$ but it is possesses a small values of Electric Quadrapole moment $(\mathrm{Q})$ due to some extent of active charge inherent to the motion and interaction inside the Nucleus.

The calculated one body density matrix element was carried by the use of OXBASH code and then the resulted output of these files had been included in another computer program to finish the calculation and produce the multipole moment.

\section{References}

[1] P. J. Brussaard and P. W. M. Glademans "Shell-model Application in Nuclear Spectroscopy" North-Holland Publishing Company, Amsterdam (1977).

[2] M. Wang, G. Audi, A. H. Wapstra, F. G. Kondev, M. MacCormick, X. Xu and B. Pfeiffer Chinese Physics C36(2012) 1603-2014; http://people.physics.anu.edu.au/ ecs103/chart/

[3] Http://ie.lbl.gov/education/isotopes.htm, "The Berkeley Laboratory Isotopes Project's, Exploring the Table of Isotopes, Last updated May 22, 2000, Richard B. Firestone, e-mail: rbf@lbl.gov.”.

[4] Z. Wang and Z. Ren, Phys. Rev., Phys. Rev. C, vol. 71, 9 (2005).

[5] O. V. Bespalova, I. N. Boboshin, V. V. Varlamov, T. A. Ermakova, B. S. Ishkhanov, A. A. Klimochkina, S. Y. Komarov, H. Koura, E. A. Romanovsky, and T. I. Spasskaya, Bulletin of the Russian Academy of Sciences: Phys., vol. 74, no. 4, 542 (2010).

[6] B. A. Brown, J. M. Allmond, A. E. Stuchbery, A. GalindoUribarri, E. Padilla-Rodal, D. C. Radford, J. C. Batchelder, M. E. Howard, J. F. Liang, B. Manning, R. L. Varner, and C. H. Yu, Phys. Rev. C 90, 6 (2014).

[7] J. M. Yao, M. Bender, P-H. Heenen, Pys. Rev. C91, 023401(2014).

[8] G. Neyens; Rep. Prog. Phys., 66, 633 (2003).

[9] B. A. Brown, B. H. Wildenthal, C. F. Williamson, F. N. Rad, S. Kowisiki, H. crannell and J. T. O'Brien; Phys. Rev., C 324, 1127 (1985).

[10] L. R. B. Elton; "Nuclear Sizes", Oxford University Press (1961). 